\title{
Interacción y uso de tecnologías en los procesos de enseñanza y aprendizaje
}

\section{Interaction and use of technologies in teaching and learning processes}

\author{
Isabel María Gallardo Fernández ${ }^{1 *}$ \\ isabel.gallardo@uv.es, https://orcid.org/0000-0001-7505-5469 \\ Ana de Castro Calvo** \\ acastro@florida-uni.es, https://orcid.org/0000-0002-7380-8225 \\ Héctor Saiz Fernández* \\ saizfer@alumni.uv.es, https://orcid.org/0000-0002-6340-1149 \\ * Universitat de València, Grupo de Investigación CRIE, Valencia, España \\ ** Florida Universitaria, Valencia, España.
}

\section{Resumen:}

El nuevo escenario de la sociedad del conocimiento y los desafíos de aprendizaje que debe enfrentar la educación en este milenio han sido determinados por la transformación del mundo en las esferas económicas, políticas y culturales.

En este artículo analizamos los procesos de interacción que se producen en los contextos educativos cuando las tecnologías son una oportunidad para lograr cambios profundos que contribuyan a mejorar los procesos de enseñanza aprendizaje desde nuevos planteamientos pedagógicos y curriculares. Todo ello conlleva una reorganización de los centros educativos, la formación y actualización del profesorado y la colaboración de las familias. Los docentes tienen ante sí el reto de cuidar las relaciones y los ambientes que lo hacen posible.

Este trabajo se vincula con el Proyecto Escuel@ Digit@l que se inscribe en el campo de la Tecnología Educativa y específica-

\begin{abstract}
:
The new scenario opened up by knowledge society and the learning challenges that education is facing nowadays have been determined by the transformation of the world at economic, political and cultural levels.

In this article we explore the interaction and socialization processes that are taking place in learning environments when technologies are an opportunity to achieve deep changes that contribute to improving teaching-learning processes from a new pedagogical and curricular approach. All this involves a reorganization of education centres, the training and updating of the teachers and the collaboration of the families. Teachers face the challenge of taking care of the relationships and the contexts that make possible the articulation of the previously-mentioned lines of action.

This work is linked with the Project Escuel@Digit@| which is being developed in the field of Educational Technology
\end{abstract}

1 Dirección para correspondencia (correspondence address):

Isabel María Gallardo Fernández. Departamento de Didáctica y Organización Escolar. Facultad de Magisterio. Campus de Tarongers. Avda. dels Tarongers, s/n. 46022 Valencia (España). 
mente en la línea que tiene por objeto de estudio las políticas y las prácticas para la innovación e integración pedagógica de las TIC en el sistema educativo. Basándonos en los análisis y entrevistas realizadas como parte del desarrollo del Proyecto, presentamos algunas evidencias de la investigación acometida que muestran que únicamente la sustitución de un tipo de tecnología por otra no transforma la forma de enseñar ni los procesos para aprender en la sociedad del siglo XXI. El desarrollo y transferencia de habilidades, destrezas, conocimientos y actitudes se da desde el diálogo que se produce en la interacción entre docentes y alumnado lo que conlleva un universo de significados.

\section{Palabras clave:}

Tecnología; interacción; formación del profesorado; innovación; contextos educativos.

\section{Résumé :}

Le nouveau scénario de la société de la connaissance et les défis d'apprentissage auxquels l'éducation doit faire face en ce millénaire ont été déterminés par la transformation du monde dans les domaines économiques, politiques et culturels.

Dans cet article, nous analysons les processus d'interaction qui se produisent dans les contextes éducatifs lorsque les technologies sont l'occasion de réaliser des changements profonds qui contribuent à améliorer les processus d'enseignement-apprentissage à partir de nouvelles approches pédagogiques et curriculaires. Tout cela entraîne une réorganisation des centres éducatifs, la formation et la mise à jour du personnel enseignant et la collaboration des familles. Les enseignants font face au défi de prendre soin des relations et des environnements qui le rendent possible.

Ce travail est lié au projet Escuel@ Digit@l qui fait partie du domaine de la technologie éducative et spécifiquement qui a pour objet d'étude les politiques et pratiques pour I'innovation et l'intégration pédagogique des TIC dans le système éducatif. Sur la base des analyses et des entrevues menées dans le cadre du développement du projet, nous présentons les preuves de recherches menées qui montrent que seule la substitution d'un type de technologie de l'autre ne transforme pas la façon d'enseigner et d'apprendre les processus dans la société du siècle XXI. Le développement et le transfert des compétences, des connaissances et des attitudes sont donnés par le dialogue qui se produit dans l'interaction entre les enseignants et les étudiants, ce qui implique un univers de significations.

\section{Mots clés:}

Technologie; interaction; la formation des enseignants; l'innovation; contextes éducatifs
Fecha de recepción: 2-5-2018
Fecha de aceptación: 9-10-2018 


\section{Introducción. Interacción y uso de las tecnologías en Educación Primaria}

En este artículo pretendemos realizar una aproximación al sentido y significado de los procesos de interacción y uso de las tecnologías en Educación Primaria en el marco del proyecto de investigación Escuel@ Digital: análisis y propuestas para la producción y uso de los contenidos digitales educativos (EDU2015-64593-R). Trataremos igualmente de justificar brevemente cuáles han sido los motivos que nos han Ilevado en nuestro proyecto de investigación a analizar el estado actual de la producción, distribución y utilización pedagógica en las aulas de los contenidos digitales destinados a Educación Primaria. A continuación, explicitamos cuáles son algunas de las razones que justifican la necesidad de analizar las interacciones que se producen en la dinámica de las aulas de Primaria.

Tomando como punto de partida el enfoque de la psicología social y el de la sociología fenomenológica se considera la interacción como la base para construir la vida social, siendo además, lo que nos permite descubrir al otro en tanto que la interacción se realiza siempre con otro distinto a uno mismo. Mediante estos procesos, el sujeto adquiere capacidad reflexiva para verse a sí mismo y otorgar sentido al mundo que le rodea (Rizo, 2005). En los procesos de socialización, la interacción está íntimamente relacionada con la comunicación en los contextos educativos, y es así como los actores involucrados construyen sus discursos (Herrero, 2012; Van Manen, 2010). En el momento actual, la concepción tradicional de comunicación entendida como el sistema compuesto por receptor, emisor y mensaje, traducida al contexto educativo como estudiante, maestro y contenidos respectivamente, queda obsoleta y fuera de la dinámica de aula donde los roles se intercambian y todos los implicados tienen voz y participan de su proceso educativo. El desarrollo y transferencia de habilidades, destrezas, conocimientos y actitudes se da a través del diálogo que se produce en la interacción entre docentes y estudiantes, al igual que la evaluación del proceso que se desarrolla a través de procedimientos comunicacionales entre todos los involucrados en la tarea. Cada vez más, y potenciado por la tecnología, el conocimiento es compartido y construido de manera colaborativa. Según Lomas (2002, p. 15) "...hoy sabemos que las personas aprendemos en interacción con otras personas, con los objetos, con los textos, con el 
enᄀ- torno físico y social, (...) y así vamos construyendo las teorías del mundo que confieren sentido a nuestra existencia".

Asumimos con Bruner (1997) que educar es provocar y facilitar situaciones de relación que proporcionen al alumnado posibilidades de vivir y experimentar el valor y el placer de descubrir juntos, de aprender juntos, de compartir y de cooperar con los iguales y también con los adultos. Así el aula se convierte en escenario comunicativo donde se fomentan procesos de interacción que facilitan la adecuada socialización (Álvarez, 1990; Gallardo, 2015; Mercer, 1997; Schaffer, 1989). Para mejorar los procesos de enseñanza y aprendizaje, la curiosidad, la emoción, la empatía o los mecanismos de atención han de ser elementos claves "que nos abren caminos, que nos mueven y nos dan razones para anhelar y buscar realidades que creemos mejores que lo que nos rodea" (Gimeno, 2010, p. 13).

En 2015 la Asamblea General de las Naciones Unidas aprobó la Agenda 2030 para el Desarrollo Sostenible en cuyo proceso de construcción se valoró una buena educación como una prioridad para la acción junto a una mejor atención de la salud. Siendo coherentes con el momento de desarrollo humano en el que nos encontramos, los Objetivos de Desarrollo Sostenible, y más concretamente aquellos puntos referidos a los procesos educativos, centran su intención en el desarrollo de habilidades metacognitivas, creativas y relacionales. Resulta prioritario situar el aprendizaje en un contexto de desarrollo global e integrado en las problemáticas medioambientales, económicas y sociales (UNESCO, 2017). Lo pedagógico ha de apostar por la capacidad creativa y el desarrollo crítico de los estudiantes como medio para acercarnos a un desarrollo social inclusivo y sostenible. En este marco de desarrollo, las Tecnologías de la Información y la Comunicación (TIC) hacen posible que los estudiantes accedan a la información a nivel global y facilita que ésta pueda gestionarse desde un enfoque social y comunitario con un componente experiencial destacado. Además, las tecnologías permiten a los estudiantes tener a su disposición un conjunto de herramientas y recursos que crean un ambiente más dinámico donde el aprendizaje es más interactivo y significativo (Imbernón, Silva y Guzmán, 2011).

Se trata de propiciar situaciones de conversación como creación de sentido y de conocimiento en quienes aprenden. Dentro de las posibilidades que ofrecen las TIC juega un papel básico la labor e implicación del docente en el proceso de planificación de objetivos y desarrollo de 
las tareas educativas. De su labor depende el grado en que las TIC facilitan el desarrollo del conjunto de competencias clave y de su relación con el propio momento y contexto de desarrollo del estudiante. Hoy más que nunca, los docentes nos encontramos ante el reto de asumir y gestionar el cruce de culturas que se produce en la institución escolar dada la complejidad de la realidad y la diversidad del alumnado.

La competencia digital resulta cada vez más necesaria para poder participar de forma significativa en la nueva sociedad y economía del conocimiento del siglo XXI (INTEF, 2017). De ahí la necesidad de comenzar a trabajar desde una perspectiva inclusiva de la competencia digital, es decir, trabajar inmersos en la tecnología, planificar desde el contexto tecnológico y ser críticos con los procesos de uso de la tecnología que desarrollamos en los centros educativos.

En este artículo presentamos, en primer lugar, el estado de la cuestión en torno al uso de las TIC en los contextos educativos actuales, su relación con los cambios metodológicos en la acción docente y el desarrollo de las competencias clave; específicamente, la competencia digital en los estudiantes. Una vez contextualizado el tema, hacemos referencia a los componentes metodológicos propios del Proyecto que han de ser tenidos en cuenta para entenderlo en su amplitud. Esto da paso a la exposición de algunos de los resultados surgidos en la investigación en relación con la interacción entre tecnología y miembros de la comunidad educativa de la etapa de Educación Primaria. Por último, compartimos una serie de conclusiones y dejamos constancia de las posibles líneas de investigación complementarias a este Proyecto.

\section{Marco teórico: estado de la cuestión}

En esta última década las tecnologías digitales han penetrado en los centros educativos del contexto español, impulsadas entre otros factores, por la existencia de distintas políticas públicas, tanto de ámbito nacional como autonómico (Area et al., 2014; Meneses, Fàbregues, Jacovkis y Rodríguez-Gómez, 2014). Este hecho, junto con la omnipresencia de la tecnología digital en la vida de los y las docentes y del alumnado, está provocando una alteración importante en las formas de producir, distribuir y acceder a la cultura y la información por parte de los agentes educativos. 
Para adentrarnos en el estado de la cuestión sobre el tema que nos ocupa se ha realizado una búsqueda en diferentes bases de datos y publicaciones periódicas de revistas educativas, así como de contribuciones relacionadas con la presencia y uso de recursos tecnológicos en los centros educativos. La búsqueda se centra en recabar información sobre la realidad de las TIC en los contextos educativos actuales en relación con los estudiantes, el profesorado, el contexto organizativo y las familias.

Así hemos podido comprobar como existen diferentes investigaciones relacionadas con la competencia digital y la competencia mediática de estudiantes, docentes y familias. En este sentido, Pérez-Escoda, Castro-Zubizarreta y Fandos-Igado (2016) exponen que el desarrollo de la competencia digital precisa de un proceso educativo concreto, ya que una interacción básica entre estudiante y dispositivo no garantiza el desarrollo de la misma. Además, estos autores evidencian que un mayor desarrollo de la competencia digital suele estar condicionado por el uso que se hace de lo tecnológico en contextos informales, para concluir explicitando que existe una carencia respecto al trabajo de la competencia en las aulas. En el mismo sentido, surgen investigaciones que cuestionan el grado de competencia digital y mediática de estudiantes, docentes y familias, relacionando el contexto educativo con la realidad fuera del centro y la influencia que ejercen sobre ellos y la manera de construir conocimiento sobre los medios de comunicación (AguadedGómez, 2012; Ferrés, 2013; Parola y Delgado, 2013; Ramírez-García y González-Fernández, 2016; Velez, 2017).

Asimismo, diferentes contribuciones muestran que el uso de dispositivos tecnológicos digitales influye de forma determinante en el proceso de enseñanza aprendizaje. Sánchez (2017) expone que su uso mejora significativamente el proceso de adquisición de conocimientos nuevos, lo que revierte positivamente en un rendimiento académico más deseable y beneficioso. Sin embargo, más concretamente, Alderete, Di Meglio y Formichella (2017) muestran evidencias sobre cómo en su estudio únicamente el acceso a las tecnologías en el hogar repercute positivamente en el rendimiento del estudiante, siendo no significativa la relación entre acceso a las tecnologías en el centro educativo y rendimiento académico. En estos casos se considera el criterio de acceso a la tecnología como condicionante para un mejor rendimiento. Sin embargo, no se presta suficiente atención a la dimensión docente como agente mediador entre el aprendizaje y la tecnología. 
Respecto al profesorado, investigaciones como la de Valverde, Fernández y Revuelta (2013) y De Pablos (2015) señalan que los docentes expresan mayoritariamente emociones positivas relacionadas con el uso educativo de las TIC. Por su parte, García-Valcárcel, Basilotta y López (2014) exponen como los docentes otorgan una alta potencialidad a las TIC para implementar el aprendizaje colaborativo y el desarrollo de competencias transversales de gran relevancia. En ambos casos, un tema recurrente son las dificultades a las que se enfrenta el profesorado con el uso educativo de las TIC. Del mismo modo, un hecho constatado en las investigaciones realizadas es que la introducción de las TIC en el aula no conlleva necesariamente un cambio metodológico. Por tanto, los dispositivos se utilizan como apoyos para realizar las mismas tareas académicas que antes de su uso (Fernández y Fernández, 2016; Madail, Martínez y Ramírez, 2016).

En relación con las familias diferentes estudios exponen que el nivel educativo de los hijos e hijas es el factor más influyente para el desarroIlo de habilidades y del pensamiento crítico (Sánchez-Valle, de FrutosTorres y Vázquez, 2017), así como la necesidad de un desarrollo de la competencia digital en las familias y a su vez mecanismos de colaboración entre ésta y el centro educativo (Bartau-Rojas, Aierbe-Barandiaran y Oregui-González, 2018).

Una vez presentado el marco teórico, a continuación, hacemos referencia a la propia investigación desarrollada, al Proyecto Escuel@ Digit@l, con el fin de definir aspectos metodológicos y contextuales relevantes que han de ser tenidos en cuenta.

\section{La investigación empírica}

Este estudio se sitúa en el marco de la investigación titulada, Escuel@ Digital, la escuela de la sociedad digital: análisis y propuestas para la producción y uso de los contenidos digitales educativos, en el que participan tres equipos de investigación de tres comunidades autónomas distintas. Su objetivo es analizar el estado actual de la producción, distribución y utilización pedagógica en las aulas de $5^{\circ}$ y $6^{\circ}$ de Educación Primaria de los contenidos digitales educativos o materiales didácticos online destinados a la educación en una muestra de tres comunidades autónomas de España (Canarias, Galicia y Valencia) (Area, 2015, p. 1). 
Partimos de la hipótesis de que "Ios materiales digitales permiten que los docentes puedan personalizarlos y adaptarlos a las características de su alumnado creando entornos de aprendizaje más enriquecidos y variados; en definitiva, los materiales digitales articulan nuevas formas de interacción de los aprendices con el conocimiento" (Area, 2015, p. 2).

El proyecto plantea un total de 4 estudios, cada uno con objetivos específicos. En este trabajo presentamos algunos de los resultados obtenidos en el Estudio 1 y 2, los cuales otorgan una relevancia destacada a la relación entre los cambios que se producen a nivel metodológico, organizacional y relacional en el contexto educativo con el grado en que las TIC contribuyen al desarrollo pleno del estudiante.

El desarrollo de la investigación empírica del Estudio 1 y 2 se realizó desde un planteamiento metodológico mixto por el que se usaron diferentes instrumentos para alcanzar los objetivos específicos planteados en las dos fases del estudio. La investigación se desarrolla desde un enfoque multimétodo, ya que concede una mayor flexibilidad para adaptarse a las demandas de explicación y comprensión de un contexto complejo como el actual (Ruiz, 2008). Las técnicas e instrumentos de recogida de datos, principalmente de corte cualitativo, se han construido desde el marco teórico y la experiencia previa, con el fin de que la investigación sea precisa y capaz de generar conocimiento específico sobre el tema que nos ocupa.

\section{Contexto de la investigación}

En el Estudio 1 se han analizado las características pedagógicas y tecnológicas de una muestra de repositorios y plataformas de contenidos educativos digitales actualmente existentes en nuestro país destinados a Educación Obligatoria, tanto de plataformas de contenidos educativos comerciales como de repositorios institucionales públicos. En esta primera fase del Proyecto nuestro interés se ha centrado en explorar y conocer cuál es el mapa de la oferta de materiales y recursos educativos online en el contexto español. Los resultados de investigación muestran que se detectan escasos indicios de estrategias innovadoras en los materiales didácticos analizados y que se sigue sin utilizar todo el potencial de las TIC en la concepción y utilización de los materiales (Cepeda, Gallardo y Rodríguez, 2017). En el marco de la educación inclusiva y la 
Educación para el Desarrollo Sostenible (EDS), las pedagogías activas y participativas basadas en proyectos son un ejemplo de lo que suponen las prácticas educativas de calidad propias de dichas estrategias innovadoras.

El Estudio 2 se ha centrado en identificar las visiones y opiniones sobre el tránsito de los libros de texto a los contenidos digitales educativos por parte de los distintos sectores implicados: profesorado, alumnado, familias y empresas editoriales (Peirats, Gallardo, San Martín y Cortés, 2015). El objetivo consiste en investigar sobre el impacto que estos nuevos materiales tienen sobre las visiones de los agentes educativos y sobre las prácticas de enseñanza y aprendizaje desarrolladas en los centros. En este contexto se cuestiona la reorganización de los centros, la formación del profesorado y la implicación de las familias. Hemos de tener en cuenta que el cambio del libro físico al digital implica una restructuración de recursos, espacios y metodologías que generalmente asume el profesorado, especialmente la figura del Coordinador TIC. Si el cambio al formato digital ya supone un hecho destacado para la organización educativa, este es más acusado cuando los centros deciden implementar la tecnología además de como sustituto del libro, como herramienta de trabajo diario de los estudiantes. En este último caso, las evidencias del Estudio 2 sobre un estudio de caso en un centro público que utiliza Tabletas como herramienta didáctica, muestran como los cambios acometidos se producen a diferentes niveles y relacionados con: la planificación de la enseñanza, la implicación del alumnado, la relación entre escuela y familias, el tipo de innovación y la formación del profesorado.

\section{Los instrumentos y proceso de análisis}

Respecto al Estudio 1, en el análisis de las plataformas y materiales didácticos digitales, se elaboró y validó un instrumento de análisis de datos que permitiera su aplicación a diferentes plataformas web comerciales y repositorios institucionales, así como a los materiales didácticos digitales alojados en estas plataformas. Posteriormente, se realizó un análisis comparativo entre las diferentes plataformas y materiales a partir de los resultados obtenidos construyendo un conjunto de matrices como método de triangulación de los datos.

En el Estudio 2 hemos optado por la entrevista semiestructurada, la ob- 
servación, la formulación de preguntas y la revisión documental, como técnicas de recogida de información sobre el uso de las tecnologías y la interacción en los procesos de enseñanza aprendizaje en Educación Primaria. En este sentido, hay que destacar el valor de las preguntas para relacionar la teoría y la práctica y para mostrar a los participantes del Proyecto, y en un contexto educativo al alumnado, tanto los temas de trabajo como las relaciones que existen entre ellos. Las preguntas como experiencia de formación hacen posible la construcción de aprendizajes al permitir la confrontación, discusión y formación en la dinámica y la interacción que se genera en el espacio/ambiente de aprendizaje. En el Estudio 2 también se realizó el análisis posterior de los resultados siguiendo el mismo procedimiento que en el Estudio 1.

Consideramos que la labor educativa ha de sostenerse sobre métodos de investigación cualitativa, en tanto que se plantea el proceso de investigación como la búsqueda de significado para un contexto y realidad específica que atiende a la diversidad característica de las personas. Asumimos con Angrosino (2012) que la investigación cualitativa es un arte por su flexibilidad a la hora de conducir un proceso de investigación, pues nos permite tomar unas decisiones y emplear unos instrumentos en función de aquello que queremos observar (Gibbs, 2012; Taylor y Bogdan, 1987). No obstante, ponemos en relieve todo método de investigación que nos ayude a dar significado a nuestro trabajo, dando valor, como hemos dicho anteriormente, a la perspectiva multimétodo.

A continuación, presentamos algunas evidencias a modo de resultados que emanan de la investigación llevada a cabo, concretamente del Estudio 1 y 2 y de las entrevistas realizadas al profesorado.

\section{Análisis de resultados}

En este Proyecto la mejora e innovación docente se convierte en objeto de estudio e investigación en las aulas de Educación Primaria, ya que se considera relevante analizar cómo el docente gestiona y planifica los diferentes proyectos y tareas que requieren del uso de la tecnología en los procesos de enseñanza aprendizaje.

Los resultados que presentamos surgen del proceso de análisis y triangulación que se ha realizado respecto al Estudio 1 y 2. En el primer caso, el instrumento elaborado para el análisis de las plataformas y materiales, 
dio lugar a un conjunto de información sobre la que se realizó una triangulación temporal, comprobando la constancia de los resultados en un momento definido determinado, y espacial, revisando las coincidencias entre las diferentes plataformas y portales analizados. Como producto, se obtuvieron primeramente un conjunto de matrices (nivel 1) sobre las que se realizó nuevamente un análisis comparativo y que dio lugar a otro grupo de matrices de mayor concreción (nivel 2). Las categorías que forman las matrices son definidas a partir de los fundamentos teóricos y en relación a los objetivos específicos de cada etapa del Proyecto. A partir de las matrices resultantes se realizó un informe preciso sobre las conclusiones obtenidas.

En el caso del Estudio 2, se diseñaron y construyeron los diferentes instrumentos que nos sirven para realizar la investigación en los centros: guion de entrevistas, plantillas de observación y fichas sobre los recursos tecnológicos del centro. Una vez realizado el trabajo de campo y recogidos los datos, se realizó un análisis de contenido de los mismos y se siguió el protocolo antes mencionado para el Estudio 1: triangulación de datos, creación de matrices (niveles de concreción 1 y 2) y realización del informe final. A continuación, presentamos algunos resultados fundamentales de la investigación desarrollada a partir de las conclusiones recogidas en los informes anteriormente señalados.

En relación con el Estudio 1 y 2 cabe destacar que las evidencias recogidas en las entrevistas realizadas muestran que el profesorado que utiliza materiales didácticos digitales rara vez emplea recursos ya creados, de repositorios o plataformas editoriales, como base de trabajo en su tarea diaria. Generalmente, los docentes entrevistados hacen referencia a su existencia y a su valor como complemento para su trabajo, pero consideran preciso adaptar estos materiales en la mayoría de los casos. Los docentes prefieren construir los materiales ellos mismos para poder responder de forma más precisa a las necesidades del grupo-clase en unidades didácticas organizadas desde el diseño de tareas y proyectos (López, Peirats, Gallardo y Cortés, 2014).

En base a las evidencias emanadas de las entrevistas al profesorado, tal y como hemos observado en las aulas de $5^{\circ}$ y $6^{\circ}$ de Educación Primaria, la dinámica de aula se basa en la potenciación de interacciones significativas para los estudiantes, y constatamos que éstas hoy en día pueden darse personalmente entre dos estudiantes, de forma virtual en plataformas colaborativas o incluso con materiales digitales que permi- 
ten la interacción a mayor o menor grado. Un ejemplo de ello es Google Classroom, el cual nos permite utilizar un gran número de herramientas para almacenar y compartir contenidos, gestionar tareas, comunicarse entre alumnos, docentes y familias, obtener informes personalizados, entre otras. En concreto, la maestra de la clase de quinto de Primaria comenta que esta plataforma facilita mucho la labor de planificación a la hora de utilizar recursos de forma integrada con otras plataformas como YouTube.

De las entrevistas y observaciones realizadas, podemos inferir que las interacciones básicas que se producen entre alumnado y docentes en un contexto mediado por la tecnología, se producen de forma diferente a como se podían dar años atrás. La tecnología y la red de información a la que nos permite acceder, constituyen un espacio demasiado amplio y sin orden, en el que el significado de la tarea puede perder valor o incluso perderse entre los diferentes contenidos existentes. Por tanto, los docentes han de demostrar en este sentido una competencia suficiente para poder organizar las tareas y contenidos dentro de este espacio virtual para que la interacción producida conlleve resultados significativos y relevantes para los estudiantes.

En relación al docente y su responsabilidad en el proceso de enseñanza aprendizaje, cabe señalar que en un entorno tecnológico las responsabilidades atribuidas al docente aumentan, ya que esto supone desde la implementación de los dispositivos a la labor de organización y planificación de las unidades de contenidos.

De forma reiterada los docentes resaltan la casi inexistente oferta formativa que se realiza desde la Administración Educativa. Los programas de formación existentes suelen centrarse en buenas prácticas. Sin embargo, la mayoría de docentes entrevistados argumentan que su manera de formarse en el uso educativo de las tecnologías ha sido en el aula con sus estudiantes y básicamente, en un modelo de ensayo error. Asimismo, atribuyen un componente significativo y de gran valor a la autoformación que ellos realizan en el día a día, y que es lo que posibilita el aprendizaje colaborativo a nivel de centro.

Además de los procesos de formación a nivel de aula, el profesorado entrevistado también se refiere a cómo la tecnología modifica la estructura de la organización del centro educativo y específicamente de la organización del aula. En el primer caso, los centros se han visto influidos por nuevas plataformas de gestión de resultados académicos, asistencia 
y burocracia oficial a la que los docentes han tenido que adecuarse e incluir en su actividad diaria por requerimiento administrativo. A nivel de aula, sin embargo, los niveles de implicación son diferentes en cuanto al grado en que cada docente se compromete por el cambio que supone en su formación el uso de la tecnología. En el caso de aulas donde los dispositivos tecnológicos han sustituido al libro en formato papel, los docentes exponen como ha sido necesaria una nueva estructuración del ambiente del aula a través de plataformas digitales de forma que dé cabida al desarrollo de los contenidos curriculares. Estos sistemas de organización del aula desde plataformas virtuales, como Google Classroom, aportan también una serie de posibilidades beneficiosas a la hora de compartir contenidos, mantenerlos actualizados, construir colaborativamente tareas y otorgar más y mayor flexibilidad al estudiante en función de sus ritmos de aprendizaje y sus propios intereses. En estas situaciones de aula se trata de fomentar la creatividad y el deseo de aprender.

Así mismo, estas plataformas hacen posible la apertura de nuevos canales de comunicación entre familias y centro, ya que la mayoría de estos espacios usados por los centros cuentan con sistemas de comunicación instantánea entre estudiantes, docentes y familias. Esta situación favorece la participación activa de las familias en el proceso de desarrollo de sus hijos e hijas, ya que no solo forman parte de los resultados académicos finales, sino de todo el proceso de enseñanza aprendizaje siendo parte activa y colaborativa en las plataformas digitales.

Estos son algunos de los resultados del Estudio 1 y 2 del Proyecto Escuel@Digit@l. La investigación sigue abierta y por tanto, a medida que avancemos en el Estudio 3 y 4 surgirán nuevos resultados que requerirán análisis e interpretación.

\section{Discusión y conclusiones}

Dentro del proceso dialéctico que supone la enseñanza existen criterios esenciales que lo hacen posible. Y es que este es un proceso necesariamente relacionado con la vida de los estudiantes, el cual influye en sus capacidades para acercarse, comprender e influir en su realidad. Asumimos que todos los rasgos culturales que se dan en la práctica pedagógica han de ser comprendidos por los diferentes actores implicados en el proceso ya que la realidad social que se vive en las aulas genera 
interacciones de gran complejidad. Nos situamos bajo una perspectiva sociocultural de la educación, compartida con diversos autores, que sostiene que el conocimiento se construye de forma social. No se aprende por y para sí mismo sino para poder participar con otras personas en actividades importantes del contexto de la propia sociedad (Álvarez, 1990; Wells, 2001).

Del trabajo realizado podemos inferir que la interacción y uso de tecnología conlleva un universo de significados. La institución educativa en sí genera un cúmulo de complejas interacciones que están reguladas por una normativa explícita y por unos condicionantes implícitos que nacen de su configuración social, de su cultura, de su sentido de comunidad (Pérez Gómez, 2012).

La institución educativa crea una cultura propia que transmite normas, creencias, valores, mitos, que regulan el comportamiento de sus miembros. Ese proceso de socialización en el centro se arraiga en sus estructuras, en los materiales que usa, en la forma de organizar el espacio, en la manera de articular las relaciones, etc. Cuando nos adentramos en el entramado del aula hay que ser conscientes de nuestra posición como docentes en la relación entre teoría y práctica, distinguir entre lo que se dice y lo que realmente se hace porque a veces no coincide. El aula es un espacio de complejidad (Morin, 1994) y a su vez, también de preguntas, de conflictos, de obligaciones, de intercambios, de espera, de descubrimientos, de complicidad, de investigación y de escucha. De ahí la importancia de la reflexividad del docente ante lo que ocurre en su práctica cotidiana (Schön, 1987).

Una mayor implicación de la familia en el proceso de aprendizaje dependerá también del docente y su intención de apertura al resto de miembros de la comunidad educativa. En relación a la implicación docente Gimeno y Pérez Gómez (1992) señalan que aquello que se hace realidad en educación, las ideas y proyectos de un docente, se producen porque ellos los hacen suyos de diferentes maneras; los interpretan y los adaptan para implementarlos en el aula. Por ello se considera al docente un mediador esencial entre los ideales y las prácticas, entre los proyectos y las realidades.

Desde este planteamiento, consideramos que las prácticas docentes son una oportunidad y posibilidad para arriesgarnos a explorar la realidad, siempre en una relación creativa, viva, con las tradiciones pedagógicas disponibles (Contreras y Pérez, 2010). Asumimos que la práctica 
de la enseñanza no puede resolverse únicamente disponiendo de una serie de recursos (sean o no tecnológicos) para actuar. Necesitamos, además, cultivar una sensibilidad y una apertura hacia el encuentro con el otro, la otra y lo otro; lo que nos reclama a los docentes un aprender a aunar saber, ser y sentir para desarrollar esa atención necesaria hacia lo que la relación educativa genera y suscita.

En este sentido hemos querido hacer evidente la necesidad de una implicación docente que responda a las nuevas circunstancias metodológicas y de producción de conocimiento que caracterizan a la sociedad digital. Los dispositivos tecnológicos han estado presentes a lo largo de la historia y nos han acompañado en los procesos de enseñanza aprendizaje. Sin embargo, hoy en día su conectividad e implicación en la vida de los estudiantes hace necesario repensar la organización y la manera en la que respondemos ante la realidad de los estudiantes, sus demandas y en relación a su búsqueda personal de significado. Por esto, la labor del docente ha de ser más personalizada que nunca, potenciando la diversidad y alimentando la curiosidad y el pensamiento crítico de los estudiantes. La formación continua de los docentes es un aspecto fundamental para lograr que la integración de las TIC sea una realidad (De Pablos, 2015).

Un uso generalizado y positivo de las TIC en el proceso de enseñanza aprendizaje supone inevitablemente la continua interacción entre los agentes involucrados: docentes, estudiantes y familias. Conlleva la colaboración en un trabajo individual y también en equipo, donde los procesos de investigación y experimentación regularán la experiencia de aprendizaje, y donde los estudiantes adquieran las competencias necesarias para posicionarse de forma crítica ante lo que se le plantea y con la finalidad de convertirse en un ciudadano de pleno derecho y que participa en la construcción de su realidad social, política y cultural.

Desde nuestro punto de vista la enseñanza es un actividad ética, moral y política, lo que significa que ninguna de nuestras acciones en relación con los estudiantes, sus familias o cualquier otro agente involucrado, va a ser neutra, sino que es parte de un proceso de construcción y cambio social donde están integrados los contenidos curriculares y se trabaja alrededor de problemas reales.

La incorporación de las tecnologías en los procesos de enseñanza y de aprendizaje es un tema complejo y a día de hoy genera un debate continuo. En este artículo hemos abordado algunas de las cuestiones 
que, con toda seguridad, habremos de retomar en futuros trabajos. También consideramos que ha de ser un tema de reflexión para todos los agentes implicados en el sistema educativo y a nivel social, ya que la tecnología afecta cada vez más a todas las personas y en gran parte de momentos de su día a día. Un uso incorrecto o indebido de la misma puede acarrear consecuencias negativas y si estas se producen en la etapa de desarrollo de los estudiantes, estos cambios serán cruciales y significativos para la construcción de su identidad personal.

Tras el recorrido realizado, constatamos que siguen quedando muchas preguntas abiertas y algunas de ellas las formulamos a continuación:

¿Cómo se está potenciando el uso de las tecnologías en los contextos educativos?

¿En qué aspectos las TIC fomentan la construcción del pensamiento de los estudiantes?

¿Qué formación pedagógica tiene el profesorado en el uso real de las TIC?

¿Cómo se está evaluando el uso de las herramientas digitales en los contextos educativos?

¿En qué sentido el uso de la tecnología genera y provoca innovación docente?

¿Qué modelo pedagógico propugnan?

\section{Referencias}

Aguaded-Gómez, J. (2012). La competencia mediática, una acción educativa inaplazable. Comunicar, 20 (39), 7-8. doi: 10.3916/C39-2012-01-01

Alderete, M., Di Meglio, G., \& Formichella, M. (2017). Acceso a las TIC y rendimiento educativo: ¿Una relación potenciada por su uso? Un análisis para España. Revista de Educación, 377, 54-81. doi: 10.4438/1988-592X-RE-2017-377-353

Álvarez, A. (1990). Diseño cultural: Una aproximación ecológica a la educación desde el paradigma histórico-cultural. Infancia y aprendizaje, 51-52. doi: 10.1080/02103702.1990.10822272

Angrosino, M. (2012). Etnografía y observación participante en Investigación Cualitativa. Madrid: Morata.

Area, M. (2015). Resumen Memoria Técnica del Proyecto Escuel@ Digital (EDU201564593-R). La escuela de la sociedad digital: análisis y propuestas para la producción y uso de los contenidos digitales educativos, financiado por el Plan Estatal de I+D+i. I.P. Manuel Area (Documento oficial, difusión restringida).

Area, M., Alonso, C., Correa, J., del Moral, M., de Pablos, J., Paredes, J., Peirats, J., Sa- 
Interacción y uso de tecnologías en los procesos de enseñanza y aprendizaje isabel María Gallardo Fernández, Ana de Castro Calvo y Héctor Saiz Fernández

nabria, A., San Martín, A. \& Valverde, J. (2014). Políticas Educativas TIC en España después del Programa Escuela 2.0.: las tendencias que emergen. Revista Latinoamericana de Tecnología Educativa (RELATEC), 13 (2), 11-33. doi: 10.17398/1695288X.13.2.11

Bartau-Rojas, I., Aierbe-Barandiaran, A., \& Oregui-González, E. (2018). Mediación parental del uso de Internet en el alumnado de Primaria: creencias, estrategias y dificultades. Comunicar, 26 (54), 71-79. doi: 10.3916/C54-2018-07

Bruner, J. (1997). La educación puerta de la Cultura. Madrid: Morata.

Cepeda, O., Gallardo, I. \& Rodríguez, J. (2017). La evaluación de los materiales didácticos digitales. Revista Latinoamericana de Tecnología Educativa (RELATEC), 16 (2), 79- 95. doi: 10.17398/1695-288X.16.2.79

Contreras, J. \& Pérez de Lara, N. (comps) (2010). Investigar la experiencia educativa. Madrid: Morata.

De Pablos, J. (Coord.). (2015). Los centros educativos ante el desafío de las tecnologías digitales. Madrid: La Muralla.

Fernández, F. \& Fernández, M. (2016). Los docentes de la Generación Z y sus competencias digitales. Comunicar, 24 (46), 97-105. doi: 10.3916/C46-2016-10

Ferrés, J. (2013). La competencia mediática y emocional de los jóvenes. Revista de Estudios de Juventud, 101, 89-101.

Gallardo, I. (2015). Aprender como forma de relación en Educación Infantil. ENSAYOS, Revista de la Facultad de Educación de Albacete, 30 (2).

García-Valcárcel, A., Basilotta, V. \& López, C. (2014). Las TIC en el aprendizaje colaborativo en el aula de Primaria y Secundaria. Comunicar, 21 (42), 65-74. doi: 10.3916/ C42-2014-06

Gibbs, G. (2012). El análisis de datos cualitativos en Investigación Cualitativa. Madrid: Morata.

Gimeno, J. \& Pérez Gómez, A. (1992). Comprender y transformar la enseñanza. Madrid: Morata.

Gimeno, J. (Comp.) (2010). Saberes e incertidumbres sobre el curriculum. Madrid: Morata.

Herrero, P. (2012). La interacción comunicativa en el proceso de enseñanza-aprendizaje. Revista electrónica de investigación Docencia Creativa, 1, 138-143.

Imbernón, F., Silva, P., \& Guzmán, C. (2011). Competencias en los procesos de enseñanza-aprendizaje virtual y semipresencial. Revista Comunicar, 18 (36), 107-114. doi:10.3916/C36-2011-03-01

INTEF (2017). Marco Común de Competencia Digital. Recuperado de: https://goo.gl/ R9ia89

la exploración de la dimensión comunicológica de la interacción. Global Media Journal, Edición Iberoamericana, 2(3).

Lomas, C. (2002). El aprendizaje de la comunicación en las aulas. Barcelona: Paidós.

López, M., Peirats, J., Gallardo, I. \& Cortés, S. (2014). Análisis de la aplicación de la tableta digital en un centro de Primaria. Un estudio de caso. Trabajo presentado en XIII Congreso Internacional de Formación del Profesorado (AUFOP 2014), celebrado los días 20 a 22 de noviembre de 2014 en Santander. Recuperado de https://goo.gl/Jvibsf 
Interacción y uso de tecnologías en los procesos de enseñanza y aprendizaje isabel María Gallardo Fernández, Ana de Castro Calvo y Héctor Saiz Fernández

Madail, M., Martín-Domínguez, J. \& Ramírez, E. (2016). Análisis comparativo de las prácticas docentes con recursos TIC. Estudio de casos con profesores de Infantil, Primaria y Secundaria. Revista Latinoamericana de Tecnología Educativa. 15 (1), 11-29. doi: 10.17398/1695-288X.15.1.11

Meneses, J., Fàbregues, S., Jacovkis, J. \& Rodríguez-Gómez, D. (2014). La introducción de las TIC en el sistema educativo español (2000-2010): un análisis comparado de las políticas autonómicas desde una perspectiva multinivel. Estudios de Educación, 27, 63-90. doi: 10.15581/004.27.63-90

Mercer, N. (1997). La construcción Guiada del conocimiento. Barcelona: Paidós.

Morin, E. (1994). Introducción al pensamiento complejo. Barcelona: Gedisa.

Parola, A., \& Delgado, A. (2013). La urgencia de la competencia mediática en los programas escolares. Chasqui: Revista Latinoamericana de Comunicación, 124, 4-10.

Peirats, J., Gallardo, I., San Martín, A. \& Cortés, S. (2015). Los contenidos curriculares digitalizados: Voces y silencios en el ámbito editorial. Educatio Siglo XXI, 33 (3), 3962. doi: 10.6018/j/240801

Pérez Gómez, A. (2012). Educarse en la era digital. Madrid: Morata.

Pérez-Escoda, A., Castro-Zubizarreta, A., \& Fandos-Igado M. (2016). La competencia digital de la Generación Z: claves para su introducción curricular en la Educación Primaria. Comunicar, 24 (49), 71-80. doi: 10.3916/C49-2016-07

Ramírez-García, A. \& González-Fernández, N. (2016). Competencia mediática del profesorado y del alumnado de educación obligatoria en España. Revista Científica de Educomunicación, 49 (24), 49-58. doi: 10.3916/C49-2016-05

Rizo, M. (2005). La Psicología Social y la Sociología Fenomenológica. Apuntes teóricos para la exploración de la dimensión comunicológica de la interacción. Global Media Journal, Edición Iberoamericana, 2(3).

Ruiz, C. (2008). El enfoque multimétodo en la investigación social y educativa: una mirada desde el paradigma de la complejidad. Revista de Filosofía y Socio Política de la Educación, 8, 13-28. Recuperado de https://goo.gl/dPB5wa

Sánchez, J. (2017). Realidad Aumentada en Educación Primaria: efectos sobre el aprendizaje. Revista Latinoamericana de Tecnología Educativa, 16 (1), 72-92. doi: 10.17398/1695-288X.16.1.79

Sánchez-Valle, M., de Frutos-Torres, B., \& Vázquez, T. (2017). La influencia de los padres en la adquisición de habilidades críticas en Internet. Comunicar, 25 (53), 103-111. doi: 10.3916/C53-2017-10

Schaffer, H. (1989). Interacción y socialización. Madrid: Visor.

Schön, D. (1987). La formación de profesionales reflexivos. Hacia un nuevo diseño de la enseñanza y aprendizaje en las profesiones. Barcelona: Paidós.

Taylor, S. \& Bogdan, R. (1987). Introducción a los métodos cualitativos de investigación. Barcelona: Paidós.

UNESCO (2017). Education for people and planet: creating sustainable futures for all, Global Education Monitoring Report, 2016. Recuperado de https://goo.gl/3Fe61n

Valverde, J., Fernández, M. \& Revuelta, F. (2013). El bienestar subjetivo ante las buenas prácticas educativas con TIC: su influencia en profesorado innovador. Educación XXI, 16 (1), 255-279. doi: 10.5944/educxx1.16.1.726 
Interacción y uso de tecnologías en los procesos de enseñanza y aprendizaje isabel María Gallardo Fernández, Ana de Castro Calvo y Héctor Saiz Fernández

Van Manen, M. (2010). El tono en la enseñanza. El significado de la sensibilidad pedagógica. Barcelona: Paidós.

Velez, I. (2017). La formación en alfabetización mediática y competencia digital. Fuentes, Revista de la Facultad de Ciencias de la Educación, 19 (2), 15-19.

Wells, G. (2001). Indagación dialógica. Barcelona: Paidós. 
